\title{
Producing the Triple Aim Through a TeleWound Solution
}

\author{
Joseph F. Ebberwein and Katherine F. Piette
}

Author's note: In our 25+ years in the post-acute care provider industry, we have experienced the value of integrating telehealth and remote patient monitoring into our care delivery model, which has improved our ability to care for patients at a substantially reduced cost of care. We knew that there had to be a way to apply these learnings and telemedicine technologies to assist healthcare providers-long-term acute care hospitals, skilled nursing facilities, home health agencies, hospices, etc.- who often lack access to expert wound care, to deliver evidence-based, efficacious wound care to their patients.

In this article, the focus is on diabetes and the management of foot ulcerations and ostomy care using TeleWound management, with case studies that illustrate our success through the use of two telehealth components: synchronous, bi-directional video and a wound documentation app.

\section{Methods}

For the patient case studies described in this article, board-certified wound clinicians used synchronous, bi-directional video, with the provider in multiple post-acute care settings—skilled nursing facilities, home health agencies, and hospices.

A wound documentation app was used, which allowed the provider at the bedside to photograph the patient's wound over time. This technology enabled automatic measurement of the length, width, and area of the wound and provided for correction of the photo to show the actual color of the wound.

Foot Ulcer Case Study 
This patient was experiencing significant lower extremity neuropathy and a lack of sensation in the feet. If not treated promptly and properly this complication can result in amputation. Statistics on lower extremity amputation rates in diabetics reveal: incidence is higher among African Americans, Native Americans, and Mexican Americans than Caucasians, higher rates among males, and rates increasing with the age of the diabetic. ${ }^{1}$ Lower extremity amputations are associated with poor glycemic control, foot deformities (hammertoes, bunions, etc.), neuropathy, and peripheral artery disease.

The healthcare team worked with this patient directly through the use of the Corstrata App to monitor the dermal temperature of their feet. A temperature increase serves as an indicator of inflammation, which in turn is a precursor to the development of an ulcer. The team was able to recommend proper offloading measures including total contact casting, off-loading orthotic shoe and staying off the feet to reduce the risk of development or recurrence of an ulcer.

\section{Ostomy Case Study}

The second case relates to patients with new ostomies who have been discharged from the acute care setting. Currently, 30-day readmission rates to the hospital are as high as $29.1 \%$ for ileostomy and $19 \%$ for colostomy patients. We are able to work with these patients, whether they are being seen by a home health company, which often does not have access to a board-certified ostomy clinician, or directly with the patient and/or their caregiver. Using the video app, we are able to provide education regarding the ostomy appliance and monitor the patient's ability to care for the new stoma. Other similar education focused care transitions models (even exclusive of Telehealth functionality) showed significant reduction in readmissions for ileostomy patient dischargesreadmission rate for dehydration was $15.5 \%$ for patients without the post discharge follow-up and education, but dropped to $0 \%$ in the study group. ${ }^{2}$

\section{Discussion}

In widespread use, this technology has produced significant positive results, including reduction in wound documentation time, consistency of wound images and 
measurements over time, and timely collaboration with the provider regarding the patient's treatment plan.

As described in Methods, the use of a HIPAA-compliant (Health Insurance Portability and Accountability Act) video app, which can be accessed on any smart device, allowed clinicians to consult with the provider caring for the patient. The benefits of the wound documentation app include properly identification of the etiology of the wound, determination of the most effective treatment plan, and monitoring of the progress of the patient toward healing.

\section{Growing Problem of Diabetes and the Sequelae of Foot Ulcers}

The rapid rise in the incidence of diabetes is alarming. Lower extremity complications of this disease are a significant public health concern in both the developed and developing world. In 2012, 29.1 million Americans, or $9.3 \%$ of the population, had diabetes. ${ }^{3}$ Worldwide, 415 million people have diabetes, and this number is expected to increase to 642 million by $2014 .{ }^{4}$

In the U.S., the prevalence of foot ulcers range from $4 \%$ to $10 \%$ among persons with diabetes mellitus-an annual population-based incidence of $1.0 \%$ to $4.1 \%$, with a lifetime incidence as high as $25 \% .^{5}$

Diabetic foot ulcers frequently become infected and are a major cause of hospital admissions. ${ }^{6,7}$ They also account for more than half of non-traumatic lower limb amputations in this patient population. ${ }^{6}$ The medical and financial burden on the healthcare system is estimated up to $\$ 45,000$ per patient. ${ }^{8}$ And this does not include the psychosocial effects on quality of life due to impaired mobility and substantial loss of productivity. ${ }^{9}$

Management includes identification of risk factors, patient education, and intensive podiatric care.5,10 Each of these components lends itself to the application of telemedicine. 


\section{High Cost Burden of Ostomies}

Each year approximately 120,000 people undergo surgeries, which result in an ostomy in the U.S ${ }^{11}$ (36.1\% receive a colostomy, $32,2 \%$ receive an ileostomy, and $31.7 \%$ receive an urostomy ${ }^{12}$ accounting for the nearly 800,000 U.S. ostomates. ${ }^{11}$ Due to the aging population and accompanied rising incidence in colorectal cancer and ulcerative colitis, the number of ostomies is expected to increase at an annual rate of $3 \% .{ }^{13}$ Even though ostomy procedures are commonplace, it is reported that as many as two thirds of these patients experience a complication ${ }^{14}$ often requiring further hospitalization. Ostomy surgeries carry both 'cost' to the patient in terms of psychological issues and quality of life and 'financial costs' to the overall healthcare system.

The 2013 Healthcare Cost and Utilization Project (HCUP) publication provides an overview of 30-day all-payer, all-cause readmissions to U.S. hospitals for a range of inpatient procedures in $2010 .{ }^{15} \mathrm{It}$ presents the most comprehensive national estimates available on readmissions by procedure. That study revealed the gravity of the readmission problem for ostomy patients (Table 1).

Table 1. 30-day all-payer, all-cause readmissions to U.S. hospitals: Healthcare Cost and Utilization Project (HCUP)

\begin{tabular}{|c|c|}
\hline Ostomy & Findings \\
\hline Ileostomy & $\begin{array}{l}2^{\text {nd }} \text { most frequent readmission procedure } \\
29.1 \% \text { readmission rate }\end{array}$ \\
\hline Ileostomy & $\begin{array}{l}4^{\text {th }} \text { most frequent readmission rate } \\
24.0 \% \text { readmission rate }\end{array}$ \\
\hline Colostomy & $\begin{array}{l}13^{\text {th }} \text { most frequent readmission rate } \\
19.0 \% \text { readmission rate }\end{array}$ \\
\hline
\end{tabular}

A recent Johns Hopkins study indicates that nearly $25 \%$ of privately insured colon surgery patients are readmitted to the hospital within three months of discharge at a 
cost of approximately $\$ 9,000$ per readmission and identified this a major quality improvement project for cost reduction. ${ }^{16}$ As in the case of diabetic foot ulcers, ostomies bear both a financial and psychosocial burden, and these too may be addressed by a telemedicine solution.

Through deployment of a TeleWound program, ${ }^{17}$ providers experience a positive return on investment in multiples ways. These include access to wound expertise; which leads to a reduction in wound care supply costs upwards of $30 \%$ based on current evidencebased treatment options. ${ }^{18}$ Other area of care that benefits include a reduction in labor cost due to the decrease in the frequency of dressing changes and faster closure times, as well as decreased regulatory and legal exposure resulting from more accurate and complete wound documentation. A complete wound assessment includes the etiology and location of the wound, classification of the wound, assessment of the surrounding skin, primary treatment objectives, treatment plan, documentation and additional patient information (Figure 1). ${ }^{19}$

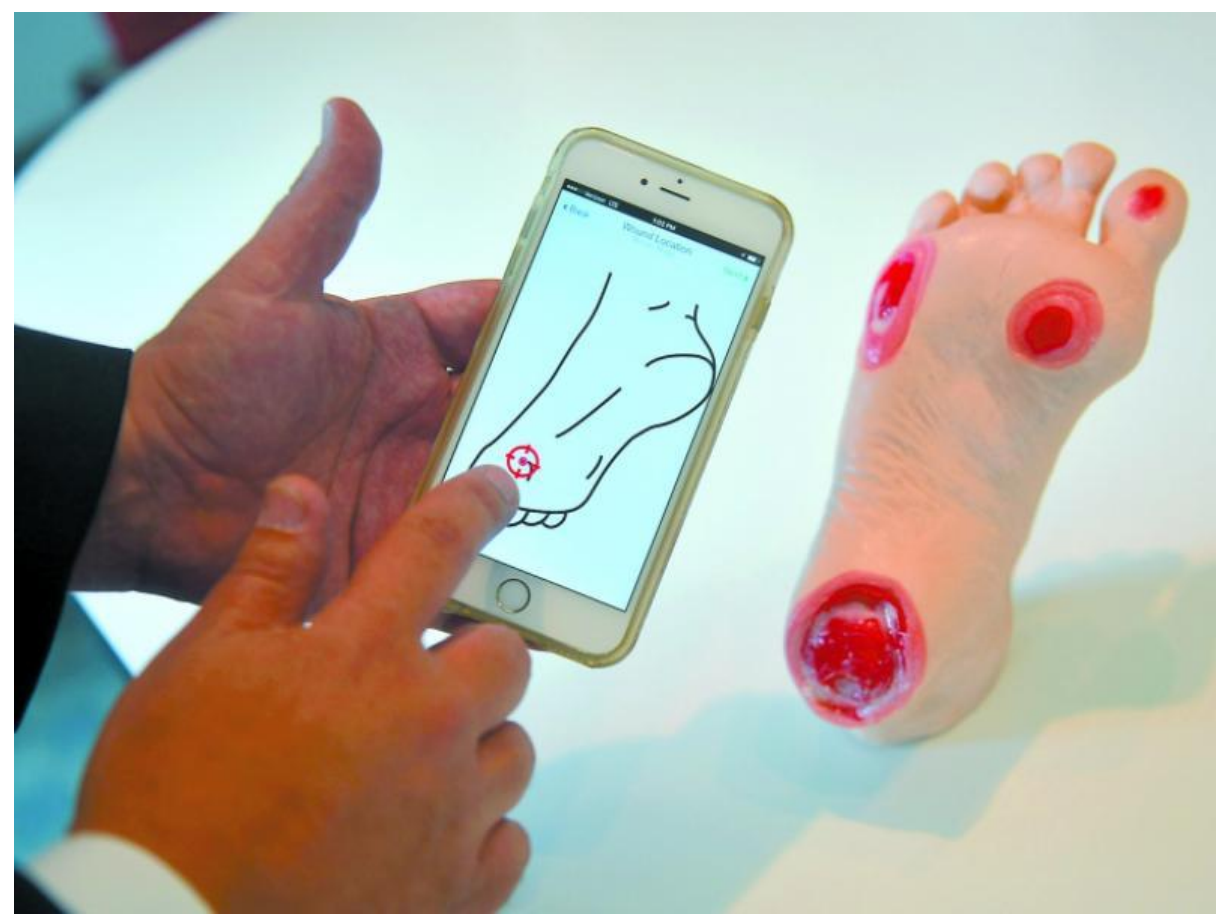

Figure 1. Illustration of the TeleWound App wound assessment 
While the current model produces cost savings for providers, the return on investment is magnified in value-based reimbursement models for providers who are in incentive sharing or risk-based contracts. In addition to return on investment as measured in dollars, our preliminary data indicate that patients and caregivers will experience increased satisfaction with their care and improved quality of life. Due to the propensity for clinical depression in patients living with chronic wounds, quality of life tools have been developed to assist providers in the care of their patients.

It is important to note that the use of the TeleWound program is not limited to the care of patients with diabetes mellitus. It is applicable to a wide variety of chronic and acute wounds, including venous ulcers, pressure injuries, cancer related tumors, and surgical complications. As value based reimbursement models evolve, care for these patients will continue to move to community and home-based models of care that leverage technology to produce better outcomes at a lower overall spend per patient.

Changes in care delivery often produce anxiety and resistance among clinicians. Clinicians may be concerned that technology is going to interfere with their ability to care for and interact with their patient. In the case of the TeleWound program, Corstrata has used this app for a year with 43 provider clinicians on 100 patients and 720 wound photo consults. We experienced that clinicians embrace the wound documentation app for ease of use and the ability of the software to auto measure the wound without having to use archaic rulers that produce inconsistent measurements. Additionally, they appreciate the ability to initiate live video consults, as they receive education about the wound and the use and application of the latest evidence based treatments. In our experience, patients and caregivers also embrace this technology as a means to help their wounds heal faster without having to leave their home.

Katherine Piette and Joseph Ebberwein are experienced leaders in the home health, hospice and private duty provider space, consulting and speaking nationally on the 
benefits of incorporating Telehealth into various value-based care delivery models across the care continuum. They are principals of Longitudinal Health, a value-based healthcare consulting firm and Founders of Corstrata, a TeleWound management company.

\section{References}

1. CDC. Diabetes Public Health Resource. 2013. URL: https://www.cdc.gov/diabetes/statistics/lea/. Accessed 12/21/16.

2. Nagle D, Pare T, Keenan E, et al. lleostomy pathway virtually eliminates readmissions for dehydration in new ostomates. Dis Colon Rectum. 2012 Dec;55(12):1266-72. doi: 10.1097/DCR.0b013e31827080c1. PubMed PMID: 23135585.

3. ADA. Statistics About Diabetes. Overall Numbers, Diabetes and Prediabetes. 2016. URL: http://www.diabetes.org/diabetes-basics/statistics. Accessed: 12/21/16.

4. International Diabetes Foundation. Diabetes: Facts and Figures. 2016. URL: http://www.idf.org/about-diabetes/facts-figures. Accessed: 12/21/16.

5. Singh N, Armstrong DG, et al. Preventing foot ulcers in patients with diabetes. JAMA. 2005;293:217-28

6. Dang CN, Boulton AJ. Changing perspectives in diabetic foot ulcer management. Int J Low Extrem Wounds. 2003;2:4-12.

7. Pinzur MS, Slovenkai MP, et al. Guidelines for diabetic foot care: recommendations endorsed by the Diabetes Committee of the American Orthopaedic Foot and Ankle Society. Foot Ankle Int. 2005;26:113-19.

8. Stockl K, Vanderplas A, et al. Costs of lower-extremity ulcers among patients with diabetes. Diabetes Care. 2004;27:2129-34.

9. Wu SC, Driver VR, Wrobel JS, Armstrong DG. Foot ulcers in the diabetic patient, prevention and treatment. Vasc Health Risk Manag. 2007;3(1):65-76. Review. PubMed PMID: 17583176; PubMed Central PMCID: PMC1994045

10. Moreland ME, Kilbourne AM, et al. Diabetes preventive care and non-traumatic lower extremity amputation rates. J Health Qual. 2004;26:12-7. 
11. Pittman J, Rawl SM, Schmidt CM, et al. Demographic and clinical factors related to ostomy complications and quality of life in veterans with an ostomy. J WOCN. 2008;35(5):493-503

12. Turnbull G. The Ostomy Files: Ostomy Statistics: The $\$ 64,000$ Question. J Ostomy Wound Management. 2003;6 Retrieved from http://www.owm.com/content/ostomy-statistics-the-64000-question. Accessed: 12/21/06.

13. Husain, SG, Cataldo, TE. Late stomal complications. Clinics in Colon and Rectal Surgery. 2008;21(1),31-40. http://doi.org/10.1055/s-2008-1055319.

14. Robertson I, Leung E, Hughes D, et al. Prospective analysis of stoma-related complications. Colorectal Dis. 2005;7:279-285.

15. Weiss AJ, Elixhauser A, Steiner C. Readmissions to U.S. Hospitals by Procedure, 2010. HCUP Statistical Brief \#154. April 2013. Agency for Healthcare Research and Quality, Rockville, MD. URL: http://www.hcupus.ahrq.gov/reports/statbriefs/sb154.pdf. Access: 12/21/16.

16. John Hopkins Medicine. Hospital Readmissions After Colon Surgery Common, Costly - and Preventable [Press Release]. 2011. URL: http://www.hopkinsmedicine.org/news/media/releases/hospital readmissions aft er colon surgery common costly and preventable. Accessed: 12/21/16.

17. Rees RS, Bashshur N. The effects of TeleWound management on use of service and financial outcomes. Telemed J E Health. 2007 Dec;13(6):663-74. PubMed PMID: 18069917.

18. Wolcott, Randall Fischenich, Vicki; Ultimate Standardization of First-Line Wound Dressings to a Single Type. Today's Wound Clinic; 2014 (April); 8(3).

19. Collier M. The elements of wound assessment. Nurs Times. 2003;99:48.

Tags diabetes, Ebberwein, foot ulcer, home health agencies, how does telehealth enhance the doctor patient relationship, ostomy, Piette, telehealth and telemedicine, telemedicine process improvement, telemedicine program implementation, telemedicine remote health, TeleWound, what is telemedicine and how does it work, wound care 
https://doi.org/10.30953/tmt.v2.52 